Case Report

\title{
Pseudotumoral and Multiple Retinal Pigment Epithelium Proliferation in Vogt-Koyanagi-Harada Disease
}

\author{
Juan B. Yepez, ${ }^{1}$ Felipe Murati, ${ }^{1}$ Michele Petitto, ${ }^{1}$ and J. Fernando Arevalo ${ }^{2}$ \\ ${ }^{1}$ Vitreoretinal Surgery Department, Clinica de Ojos, Maracaibo, Venezuela \\ ${ }^{2}$ Retina Division, Wilmer Eye Institute, School of Medicine, Johns Hopkins University, Baltimore, MD 21287, USA
}

Correspondence should be addressed to J. Fernando Arevalo; arevalojf2020@gmail.com

Received 24 July 2015; Revised 14 September 2015; Accepted 27 September 2015

Academic Editor: Shigeki Machida

Copyright ( 2015 Juan B. Yepez et al. This is an open access article distributed under the Creative Commons Attribution License, which permits unrestricted use, distribution, and reproduction in any medium, provided the original work is properly cited.

\begin{abstract}
We report a case of pseudotumoral retinal pigment epithelium (RPE) proliferation in Vogt-Koyanagi-Harada (VKH) disease, in a 50-year-old female who presented with a juxtapapillary and peripheral subretinal hyperpigmented lesions in the left eye and "sunset glow fundus," hyperpigmented striae, and multiple atrophic chorioretinal spots in the periphery. The darkly pigmented exuberant larger subretinal mass extended to the periphery with associated subretinal fibrosis. This patient demonstrated the entire clinical presentation of VKH disease, which tends to course with a chronic, bilateral, granulomatous panuveitis and exudative retinal detachment associated with poliosis, vitiligo, alopecia, and central nervous system and auditory signs. Our case is unique for the presence of exuberant, pseudotumoral RPE proliferation at the juxtapapillary region and peripheral area. Although this complication has rarely been reported, a high index of suspicion is warranted for early diagnosis and avoids unnecessary treatments of a pseudotumor.
\end{abstract}

\section{Introduction}

Vogt-Koyanagi-Harada (VKH) disease is chronic, bilateral, granulomatous panuveitis characterized by exudative retinal detachments associated with poliosis, vitiligo, alopecia, and central nervous system and auditory signs [1]. The exact cause of VKH disease remains unknown, but evidence suggests that it involves a T-lymphocyte-mediated autoimmune process directed against one or more antigens found on or associated with melanocytes. Several studies demonstrated that tyrosinase family proteins are the antigens specific to VKH disease $[2-5]$ and that $\mathrm{VKH}$ disease is characterized by a Thelper type 1 cell-mediated immune response [6,7].

Vision-threatening complications have been clearly recognized to occur in the chronic, recurrent phase of VKH disease, namely, cataract, glaucoma, subretinal neovascular membranes, and subretinal fibrosis. The occurrence of these complications is known to be associated with a worse visual outcome $[1,8,9]$. The principles of therapy of VKH disease are to suppress the initial intraocular inflammation with early and aggressive use of systemic corticosteroids, followed by slow tapering $[1,10]$. The addition of conventional immunosuppressive agents to systemic corticosteroids has been advocated $[11,12]$. Such combined treatment may shorten the duration of the disease, may prevent progression into the chronic stage, and may reduce the incidence of extraocular manifestations and complications as well.

Khairallah et al. [13] described the first case of RPE proliferation leading to subretinal fibrosis in a patient with VogtKoyanagi-Harada disease. To the best our knowledge, our case represents the second reported case of RPE proliferation leading to subretinal fibrosis in chronic VKH disease. Our case is unique in terms of the exuberant pseudotumoral retinal RPE proliferation in multiple locations at the juxtapapillary area and periphery.

\section{Case Report}

A 50-year-old woman with a 20 -year history of panuveitis associated with exudative retinal detachment in the left eye (LE) that had been treated with long-term oral corticosteroids was referred to our center. She had a juxtapapillary 


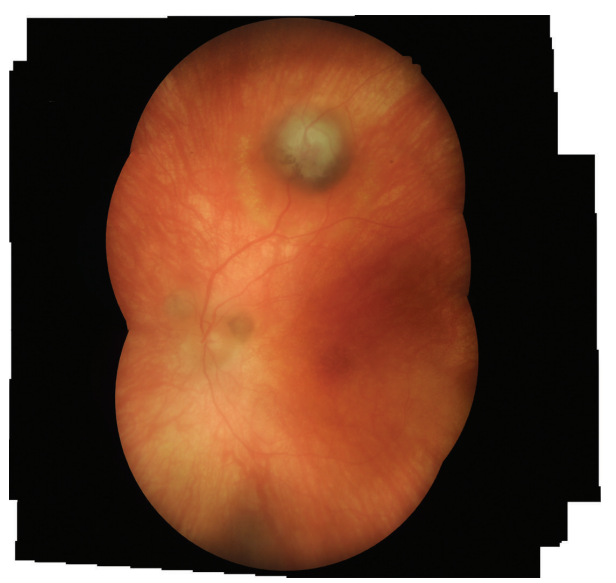

Figure 1: (A-B) Fundus examination of LE showed features of chronic recurrent Vogt-Koyanagi-Harada disease, including "sunset glow fundus," hyperpigmented striae, and multiple atrophic chorioretinal spots in the periphery (not shown); darkly pigmented exuberant subretinal mass extended to the periphery with associated subretinal fibrosis. There were 2 smaller pigmented subretinal lesions located around the optic disc.

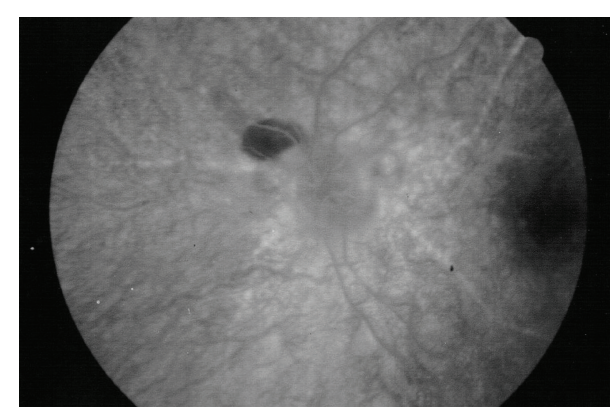

(a)

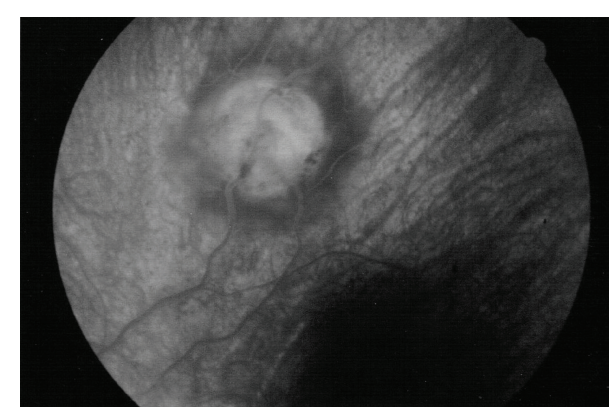

(b)

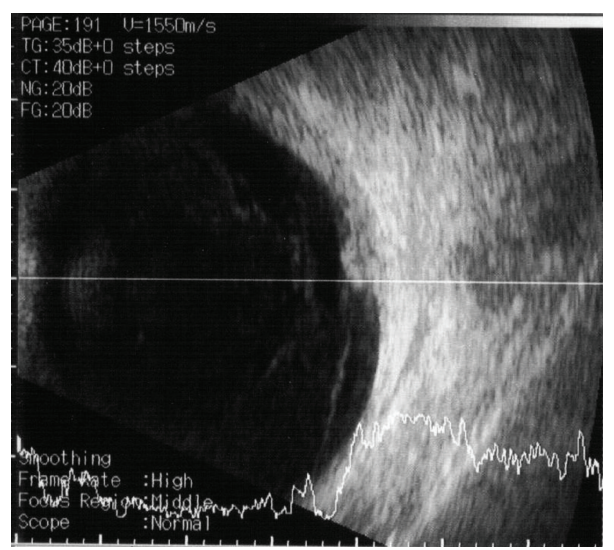

(c)

FIGURE 2: (a-b) Fluorescein angiography (FA) demonstrated background hyperfluorescence corresponding to the "sunset glow fundus" and hyper- and hypofluorescence of the RPE proliferating lesions. (c) A B-scan ultrasound of the larger peripheral RPE proliferations showed a dome-shaped mass $2 \mathrm{~mm}$ in height, high internal reflectivity, and a partial posterior vitreous detachment.

and peripheral subretinal hyperpigmented mass in LE. Her right eye had history of a chronic rhegmatogenous retinal detachment after complicated cataract surgery 5 years before presentation and no light perception visual acuity. Her visual acuity was 20/400 OS. Slit-lamp examination showed periocular vitiligo, poliosis, and posterior synechiae. There were no cells and flare in the anterior chamber or vitreous haze. Intraocular pressure was $12 \mathrm{~mm} \mathrm{Hg} \mathrm{OU}$. Fundus examination of LE showed features of chronic recurrent VKH disease, including "sunset glow fundus," hyperpigmented striae, and multiple atrophic chorioretinal spots in the periphery; a darkly pigmented exuberant subretinal mass extended to the 


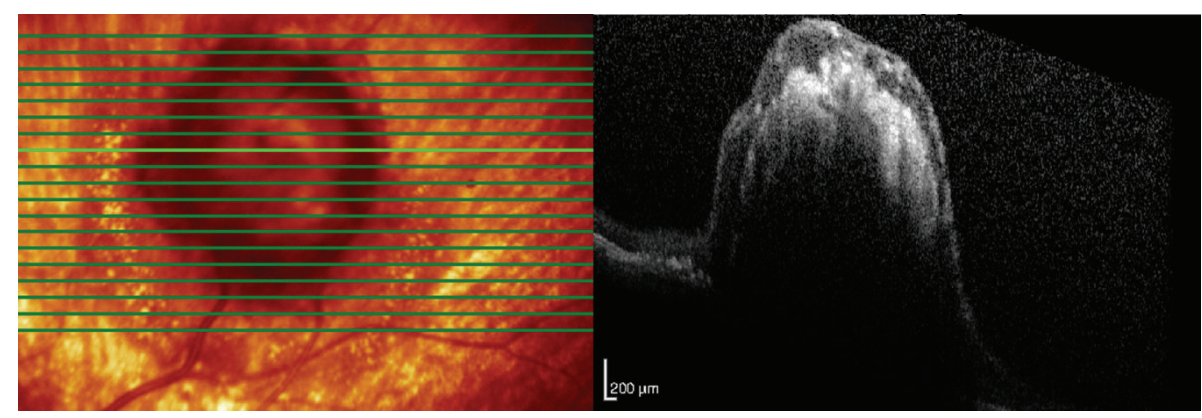

(a)

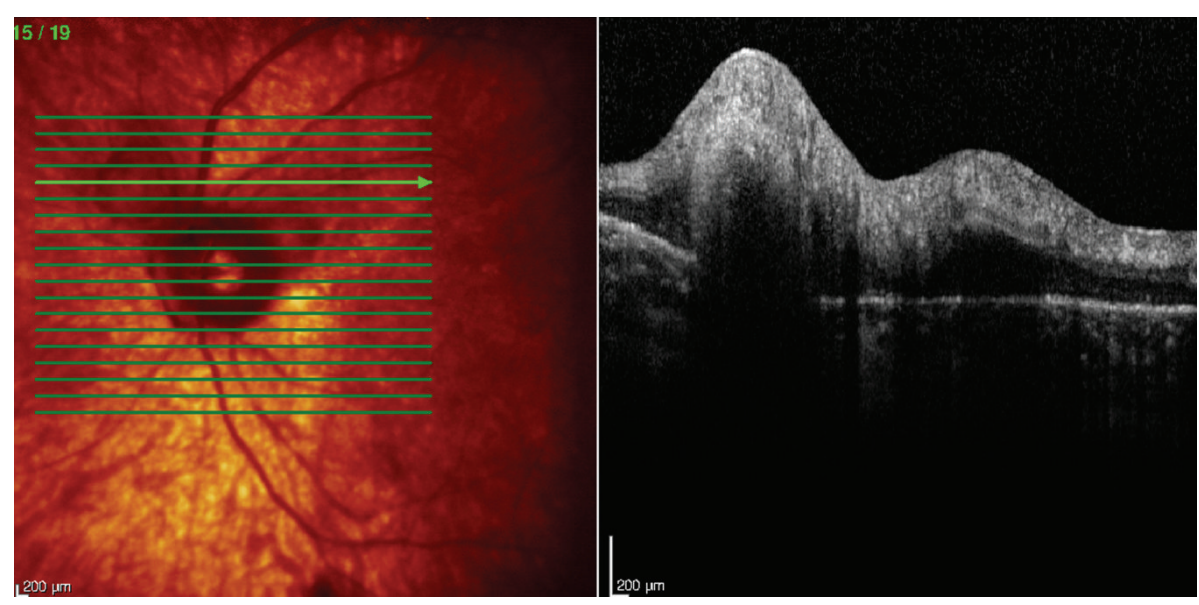

(b)

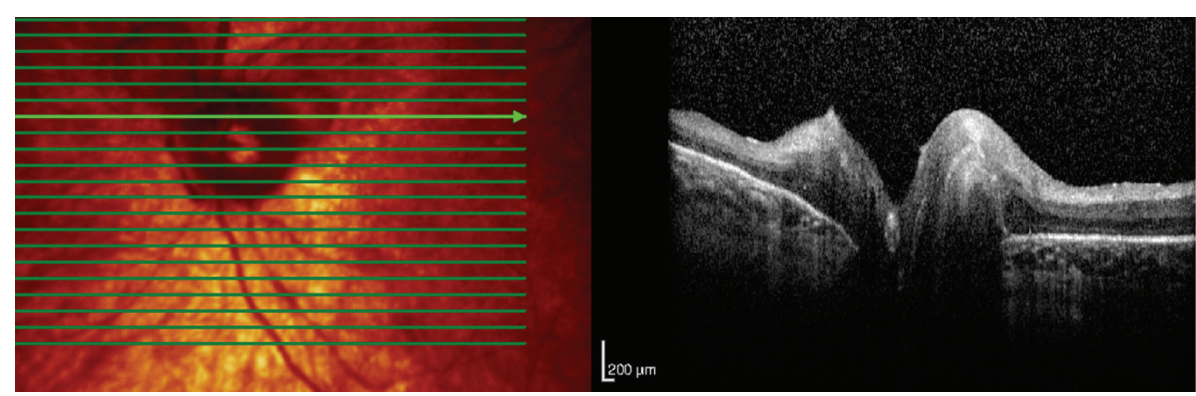

(c)

FIGURE 3: (a-c) Optical coherent tomography (OCT) in LE showed 2 lesions around the optic disc and 1 on the temporal vascular superior arcade. Overlying the dome-shaped elevation of the outer retinal layers, the choroid was thickened and the retinal pigment epithelium (RPE) was irregular.

periphery with associated subretinal fibrosis. There were 2 smaller pigmented subretinal lesions located around the optic disc (Figure 1). Fluorescein angiography (FA) demonstrated background hyperfluorescence corresponding to the "sunset glow fundus" and hyper- and hypofluorescence of the RPE proliferating lesions (Figures 2(a)-2(b)). A B-scan ultrasound of the larger peripheral RPE proliferations showed a domeshaped mass $2 \mathrm{~mm}$ in height, high internal reflectivity, and a partial posterior vitreous detachment (Figure 2(c)). Optical coherent tomography (OCT) showed 2 lesions around the optic disc and 1 on the temporal vascular superior arcade. Overlying the dome-shaped elevation of the outer retinal layers, the choroid was thickened and the retinal pigment epithelium (RPE) was irregular (Figure 3).

\section{Discussion}

Early and aggressive high-dose systemic corticosteroid therapy has become the mainstay therapy of $\mathrm{VKH}$ disease. Patients with VKH disease adequately treated with corticosteroids have a favourable visual prognosis. However, the addition of conventional immunosuppressive agents to systemic corticosteroids has been advocated to prevent the progression of $\mathrm{VKH}$ disease, lessen its duration, prevent 
severe ocular complications, and prevent systemic diseases such as ear, skin, or hair lesions [11, 12]. Our case exemplifies that complications may occur in patients with VKH disease if they are not properly treated.

The present case shows that RPE proliferation leading to subretinal fibrosis may occur in patients with $\mathrm{VKH}$ disease. The first case was described by Khairallah et al. in 2006 [13] who demonstrated that the fibrotic mass can be covered by pigmented cells. To the best of our knowledge, our case is the second described pseudotumoral RPE proliferation and adds multiple lesions including one in the peripheral retina. Although this complication has rarely been reported, a high index of suspicion is warranted for early diagnosis and avoids unnecessary treatment of a pseudotumor. In addition, we have to keep in mind the possibility that other chronic inflammatory conditions could potentially be associated with RPE proliferation and pseudotumors.

\section{Conflict of Interests}

No conflicting relationship exists for any author.

\section{References}

[1] R. S. Moorthy, H. Inomata, and N. A. Rao, "Vogt-KoyanagiHarada syndrome," Survey of Ophthalmology, vol. 39, no. 4, pp. 265-292, 1995.

[2] H. Kobayashi, T. Kokubo, M. Takahashi et al., "Tyrosinase epitope recognized by an HLA-DR-restricted T-cell line from a Vogt-Koyanagi-Harada disease patient," Immunogenetics, vol. 47, no. 5, pp. 398-403, 1998.

[3] K. Yamaki, K. Gocho, K. Hayakawa, I. Kondo, and S. Sakuragi, "Tyrosinase family proteins are antigens specific to VogtKoyanagi-Harada disease," Journal of Immunology, vol. 165, no. 12, pp. 7323-7329, 2000

[4] K. Yamaki, I. Kondo, H. Nakamura, M. Miyano, S. Konno, and S. Sakuragi, "Ocular and extraocular inflammation induced by immunization of tyrosinase related protein 1 and 2 in Lewis Rats," Experimental Eye Research, vol. 71, no. 4, pp. 361-369, 2000.

[5] K. Gocho, I. Kondo, and K. Yamaki, "Identification of autoreactive T cells in Vogt-Koyanagi-Harada disease," Investigative Ophthalmology and Visual Science, vol. 42, no. 9, pp. 2004-2009, 2001.

[6] Y. Imai, M. Sugita, S. Nakamura, S. Toriyama, and S. Ohno, "Cytokine production and helper $\mathrm{T}$ cell subsets in VogtKoyanagi-Harada's disease," Current Eye Research, vol. 22, no. 4, pp. 312-318, 2001.

[7] A. M. Abu El-Asrar, S. Struyf, F. J. Descamps et al., "Chemokines and gelatinases in the aqueous humor of patients with active uveitis," American Journal of Ophthalmology, vol. 138, no. 3, pp. 401-411, 2004.

[8] R. W. Read, A. Rechodouni, N. Butani et al., "Complications and prognostic factors in Vogt-Koyanagi-Harada disease," American Journal of Ophthalmology, vol. 131, no. 5, pp. 599-606, 2001.

[9] S. Ohno, R. Minakawa, and H. Matsuda, "Clinical studies of Vogt-Koyanagi-Harada's disease," Japanese Journal of Ophthalmology, vol. 32, no. 3, pp. 334-343, 1988.
[10] S. Ohno, D. H. Char, S. J. Kimura, and G. R. O’Connor, "VogtKoyanagi-Harada syndrome," American Journal of Ophthalmology, vol. 83, no. 5, pp. 735-740, 1977.

[11] A. M. Abu El-Asrar, M. Al Tamimi, S. Hemachandran, H. S. Al-Mezaine, A. Al-Muammar, and D. Kangave, "Prognostic factors for clinical outcomes in patients with Vogt-KoyanagiHarada disease treated with high-dose corticosteroids," Acta Ophthalmologica, vol. 91, no. 6, pp. e486-e493, 2013.

[12] A. M. Abu El-Asrar, S. Hemachandran, H. S. Al-Mezaine, D. Kangave, and A. M. Al-Muammar, "The outcomes of mycophenolate mofetil therapy combined with systemic corticosteroids in acute uveitis associated with Vogt-Koyanagi-Harada disease," Acta Ophthalmologica, vol. 90, no. 8, pp. e603-e608, 2012.

[13] M. Khairallah, N. A. Rao, S. B. Yahia, S. Zaouali, and S. Attia, "Pseudotumoral retinal pigment epithelium proliferation in a patient with Vogt-Koyanagi-Harada disease," Archives of Ophthalmology, vol. 124, no. 9, pp. 1366-1367, 2006. 


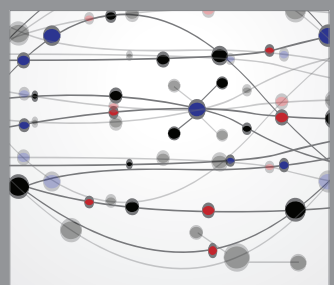

The Scientific World Journal
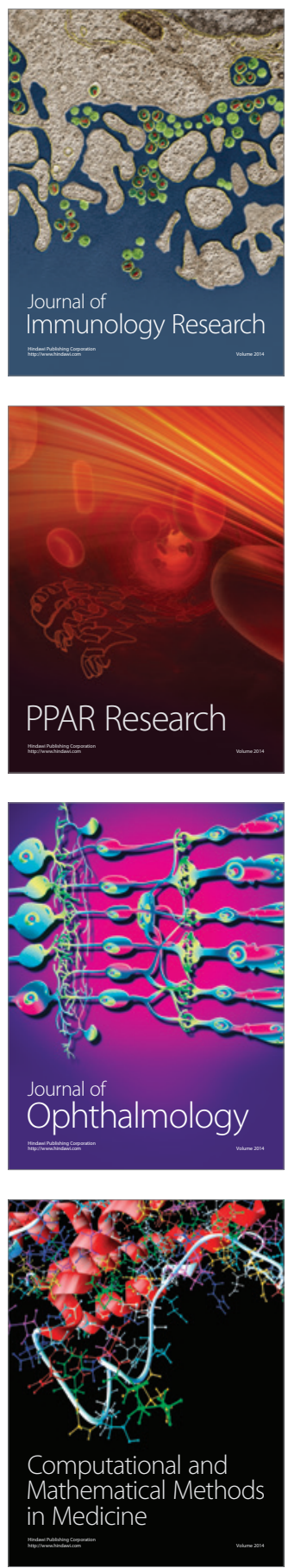

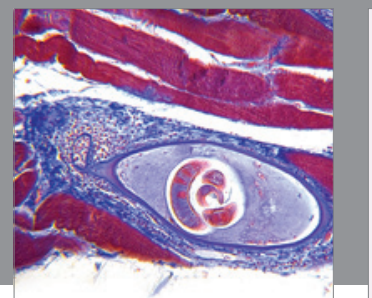

Gastroenterology

Research and Practice
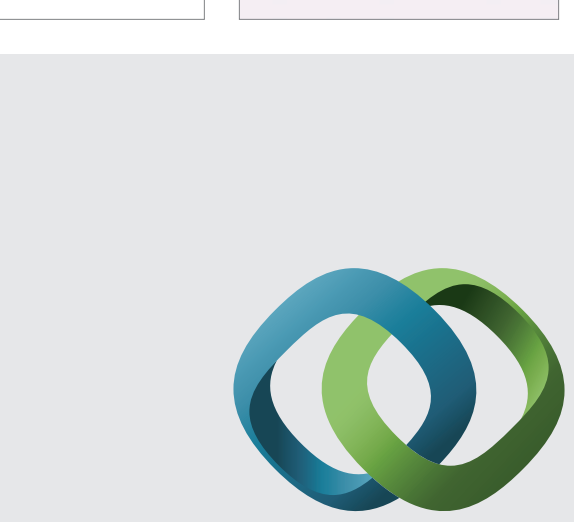

\section{Hindawi}

Submit your manuscripts at

http://www.hindawi.com
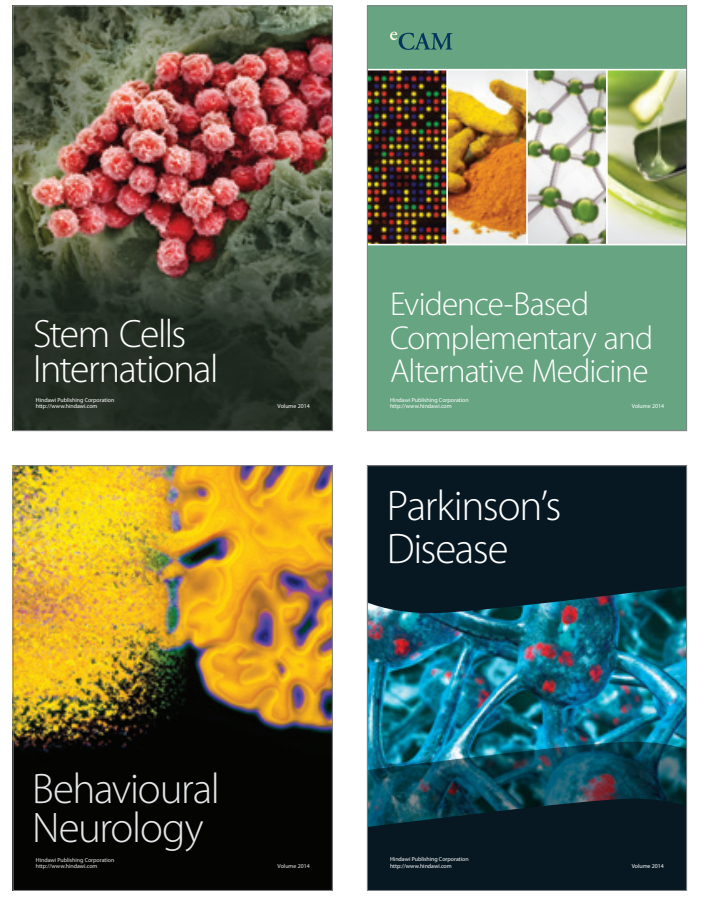
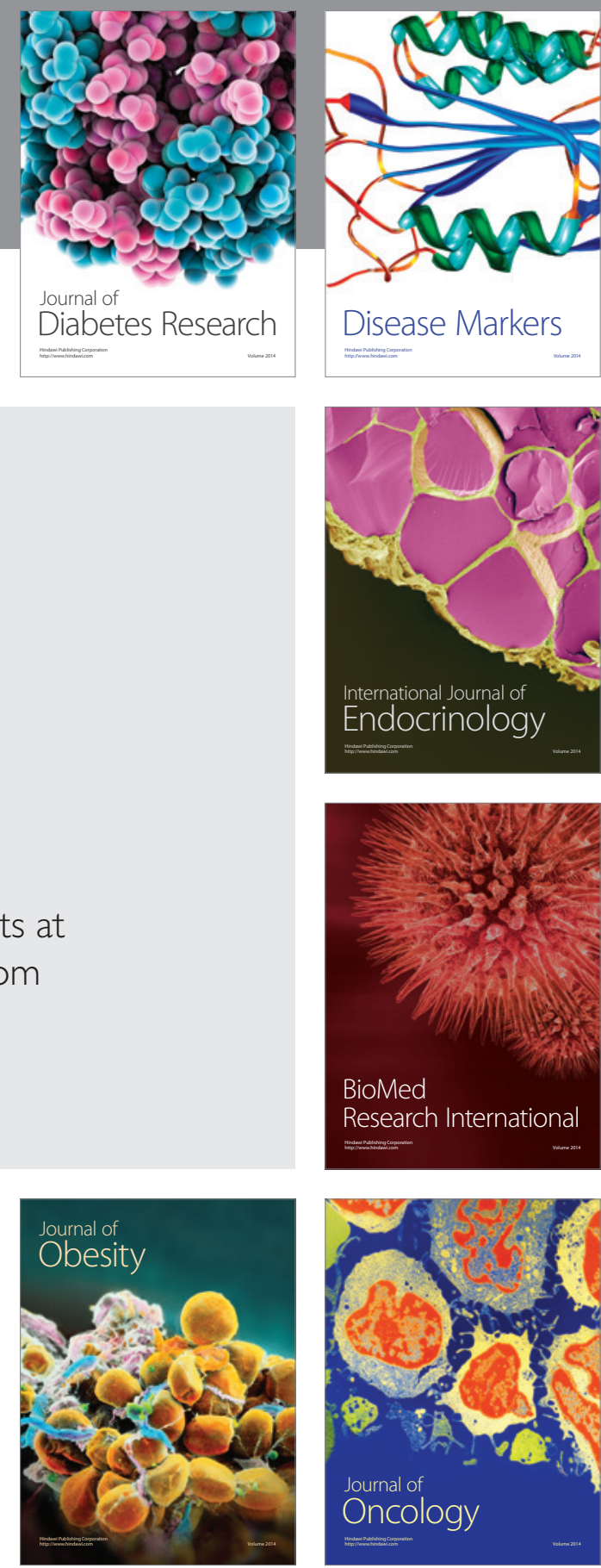

Disease Markers
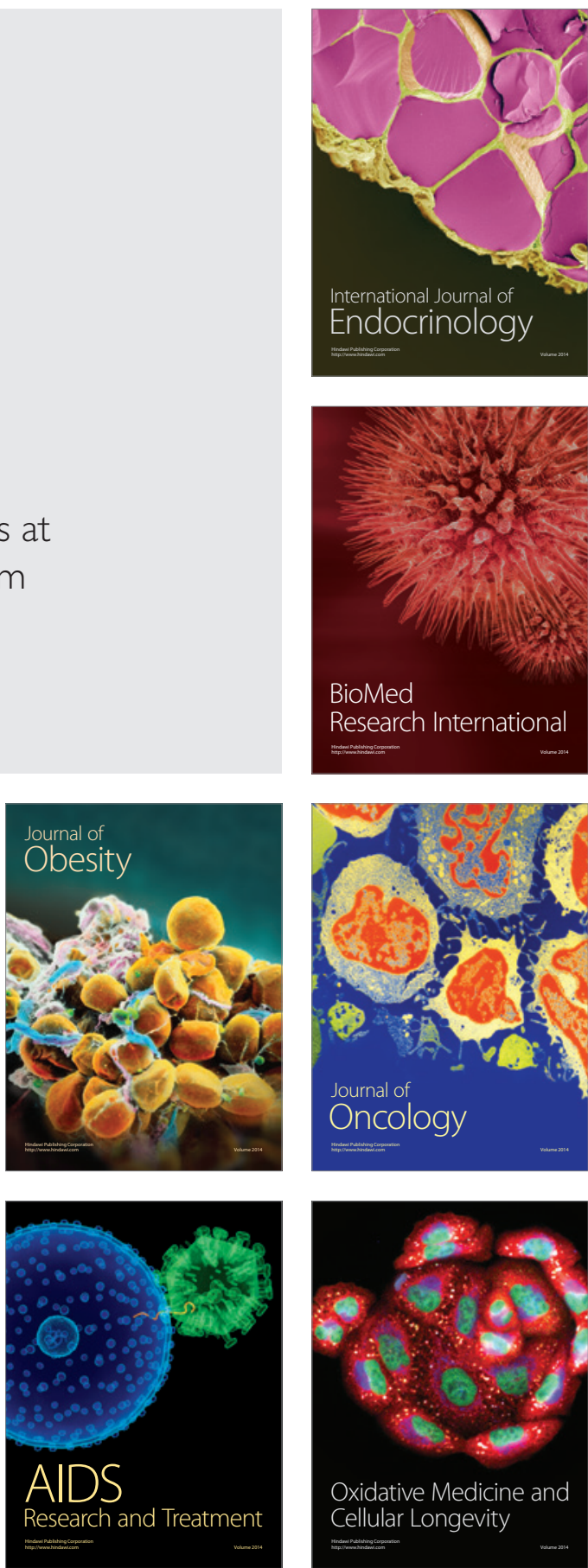